\title{
Impact strength of underground cement materials
}

\author{
Vladimir I. Travush ${ }^{1}$, Nikolay I. Karpenko ${ }^{2}$, Vladimir T. Erofeev ${ }^{3}$, Vasiliy D. Cherkasov ${ }^{3}$, \\ Denis $V$. Emelyanov ${ }^{3,{ }^{*}}$ and Irina $V$. Erofeeva ${ }^{2}$ \\ ${ }^{1}$ CJSC «Gorproekt», 105064 Moscow, Russia \\ ${ }^{2}$ Research Institute of Building Physics, 127238 Moscow, Russia \\ ${ }^{3}$ Mordovia State University, 430005 Saransk, Russia
}

\begin{abstract}
The article presents the results of a study of impact strength of underground cement materials with different water content activated by mixing water with the addition of high range plasticizers, biocidal agents, pigments, ground quartz, silica fume and fine aggregate. Samples for testing are formed in plates of size $150 \times 100 \times 10 \mathrm{~mm}$. Testing was conducted at impact drop-weight method ASTM D7136. The maximum contact force, contact duration and magnitude of the pulse of the drummer were considered as the test parameters. The good results of the studied parameters showed highly filled plasticized compositions of the new generation. The use of iron oxide with yellow and green glauconitic pigments allows obtaining good results. Activation of the mixing water led to a rise in the maximum contact force in the case of the processing mode of $\mathrm{E}+\mathrm{M}$ (3-3) while reducing the duration of the contact and the magnitude of the impulse. During the test of the biocidal composites with different biocidal preparations, it was established that the best indicators of the impact strength correspond to materials with the addition of «Teflex Antiplesen».
\end{abstract}

\section{Introduction}

During operation, the structures of buildings and constructions may be impacted by shock loads, which lead to premature destruction of the floors of buildings with heavy duty, road and airfield coatings. The materials used in underground construction, such as concrete sleepers, tunnels and other subway structures, are of particular interest. Serious danger is represented by shocks of relatively low intensity, leaving no traces on the surface of the structure, but leading to the appearance of internal defects, which can be further developed under the influence of other operational loads.

In this regard, the actual problem is the creation of cement composites capable of effectively resisting the effects of seismic and shock loads.

During the research, various compositions of non-filled (cement stone) and filled cement composites were examined: with different water-cement ratio (W/C); with activated water mixing; with the addition of super- and hyperplasticizers, biocidal additives,

\footnotetext{
* Corresponding author: emelyanoffdv@yandex.ru
} 
pigments, fillers - ground quartz and microsilica, quartz powders of various sizes. Various composites have been formed, including multi-component materials of the new generation powder-activated concrete.

To date, old-generation concrete with a 3-component composition (cement-sand-water) or 4-component (cement-sand-gravel-water) using super- and hyperplasticizers and microsilica turned into less cement-intensive concrete of the transitional generation, production of which is $97-98 \%$ of the volume of concrete.

Powder-activated concrete of the new generation is plasticized concrete with an increased content of the suspension component [1-3]. The main suspension component in concrete is plasticized highly concentrated cement slurry.

The transition to new types of concrete is due to high achievements in the field of plasticisation of concrete and mortar mixtures and the appearance of the most active pozzolanic additives - microsilica, dehydrated kaolin, and highly dispersed sol [1-4]. In recent years, the concept of the use of rheologically active monomineral and polymineral fine powders of a micrometric scale level based on ground rocks has been implemented in the production of high-quality concretes [5-7].

The combination of superplasticizers and especially hyperplasticizers on polycarboxylate and polyglycolic bases with dispersed powders makes it possible to reduce the water-cement ratio to $0.24-0.28$ and to obtain superfluid cement-mineral disperse systems and concrete mixtures [8-11].

As a result of the performed studies, the authors of $[3,4,8,9,12,13,15]$ developed powder-activated concrete with a strength class of B100-B140 with a low specific consumption of cement per unit of compressive strength not exceeding 3-6 kg / $\mathrm{MPa}$.

The formation of the structure of powdered concrete, the distinctive features of which are the higher dispersity of granular components, the increased value of the areas of the phase interface, the predominance of small and hidden cryptocrysts of neoplasms, the increased density, the increased number of physical and physico-chemical contacts per unit volume of material, creates a contradictory situation in which, on the one hand, the formed structure provides increasing compressive strength, and, on the other hand, makes it relatively more brittle, which can lead to a decrease in fracture toughness and impact strength [12-16].

To determine the impact strength of materials, the Izod and Charpy methods are used. The present studies were carried out using the ASTM D7136 method using vertical impactor manufactured by Coesfeld.

The impact test was conducted with a falling load. The sample was placed in the jig in the center of the cavity in it. They released the drummer, allowing him to freely fall on the surface of the sample. At the same time, the dependence of the force on time was recorded during the contact of the impactor with the sample continuously or at certain intervals of time. The beginning of the contact between the impactor and the sample is fixed when the value of the contact force deviates from zero. As soon as the impactor touches the surface of the sample, it bends the sample and causes a local dent, as a result, the contact force increases. Sharp jumps of contact force are explained by the processes of failure, which lead to a sudden loss of stiffness of the sample in the contact zone. The maximum force $F_{\max }$ is determined from the dependence of the contact force on time. The duration of the contact $\tau$ was determined from this dependence.

Physicomechanical indexes obtained as a result of tests of building materials on impact resistance make it possible to determine the qualitative characteristics of the elasto-viscousplastic state of materials that change over a short period of time. In this case, stress-strain state of materials during testing was estimated by the indicator of the maximum contact force at which destruction occurs. The parameter of the duration of contact between the impactor and the samples was connected with the elastic-viscous-plastic state of the 
material. The integral sum of the varying value of the force of the impactor from the time of its contact with the samples is a characteristic of the momentum of the impactor.

As objects of study, cement composites of various types were considered. Compositions of composites are given in Tables 1,2 .

Table 1. Composite Compositions.

\begin{tabular}{|c|c|c|c|c|c|c|c|c|c|c|c|c|c|c|}
\hline \multirow[b]{2}{*}{ 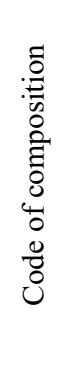 } & \multicolumn{14}{|c|}{ Content of components in parts by weight } \\
\hline & 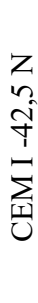 & $\begin{array}{l}\frac{\overrightarrow{0}}{\bar{\omega}} \\
\overrightarrow{3}\end{array}$ & 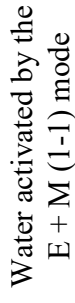 & 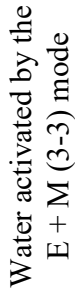 & 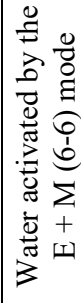 & 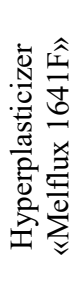 & 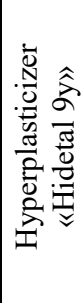 & 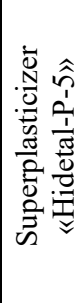 & 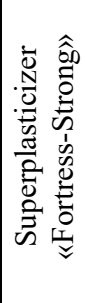 & 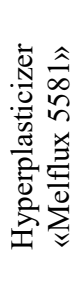 & 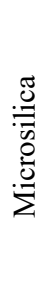 & 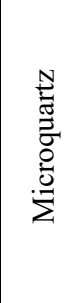 & 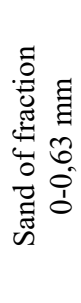 & 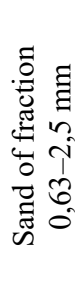 \\
\hline 1 & 1 & 0,267 & - & - & - & - & - & - & - & - & - & - & - & - \\
\hline 2 & 1 & 0,35 & - & - & - & - & - & - & - & - & - & - & - & - \\
\hline 3 & 1 & 0,171 & - & - & - & 0,009 & - & - & - & - & - & - & - & - \\
\hline 4 & 1 & 0,6 & - & - & - & 0,009 & - & - & - & - & - & 1,1 & 2,753 & 2,347 \\
\hline 5 & 1 & 0,475 & - & - & - & 0,009 & - & - & - & - & 0,1 & 0,75 & 1,775 & 1,975 \\
\hline 6 & 1 & 0,525 & - & - & - & 0,009 & - & - & - & - & - & - & 2,065 & 1,76 \\
\hline 7 & 1 & 0,56 & - & - & - & 0,009 & - & - & - & - & - & - & 2,51 & 2,14 \\
\hline 8 & 1 & 0,56 & - & - & - & & 0,012 & - & - & - & - & 0,825 & 2,065 & 1,76 \\
\hline 9 & 1 & 0,261 & - & - & - & - & - & - & 0,015 & - & - & - & - & - \\
\hline 10 & 1 & 0,289 & - & - & - & - & - & 0,015 & - & - & - & - & - & - \\
\hline M0 & 1 & 0,267 & - & - & - & - & - & - & - & - & - & - & - & - \\
\hline M1 & 1 & - & 0,264 & - & - & - & - & - & - & - & - & - & - & - \\
\hline M3 & 1 & - & - & 0,26 & - & - & - & - & - & - & - & - & - & - \\
\hline M6 & 1 & - & - & - & 0,264 & - & - & - & - & - & - & - & - & - \\
\hline
\end{tabular}

Studies and comparison of the results are performed in 5 stages according to the formulations: cement stones with different water content and with the introduction of superand hyperplasticizers (stage 1); cement composites that include sand fractions, as well as microsilica with stone flour (stage 2); cement composites for decorative purposes (stage 3); cement stones with the use of activated water activated by different regimes (stage 4); cement composites including biocide additives in their composition, as well as biocidal additives in combination with a hyperplasticizer (stage 5).

Thus, as the objects of research, materials that relate to the old, transitional and newgeneration concretes were considered [1] together with compositions with modifying structure of cement stone components, as well as formulations with additives of pigments and biocidal preparations.

During the tests on the impact strength of cement composites, the curves of the dependence of the contact force on time were obtained. An example of such a dependence for composition 1 is shown in Fig. 1. 
Table 2. Composite Compositions.

\begin{tabular}{|c|c|c|c|c|c|c|c|c|c|c|c|}
\hline \multirow[b]{2}{*}{ 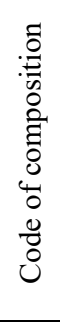 } & \multicolumn{11}{|c|}{ Content of components in parts by weight } \\
\hline & 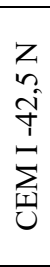 & $\begin{array}{l}\stackrel{\bar{D}}{\bar{t}} \\
\overrightarrow{3}\end{array}$ & 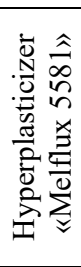 & 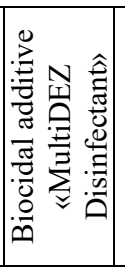 & 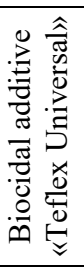 & 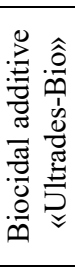 & 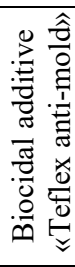 & 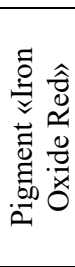 & 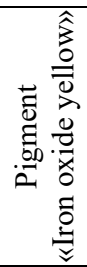 & 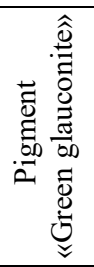 & 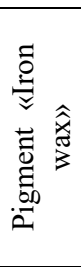 \\
\hline $\mathrm{T} 1$ & 1 & 0,267 & - & - & - & - & - & - & - & - & - \\
\hline $\mathrm{T} 2$ & 1 & 0,267 & - & 0,03 & - & - & - & - & - & - & - \\
\hline $\mathrm{T} 3$ & 1 & 0,267 & - & - & 0,03 & - & - & - & - & - & - \\
\hline $\mathrm{T} 4$ & 1 & 0,267 & - & - & - & 0,03 & - & - & - & - & - \\
\hline T5 & 1 & 0,35 & - & - & - & - & - & - & - & - & - \\
\hline T6 & 1 & 0,35 & - & 0,03 & - & - & - & - & - & - & - \\
\hline $\mathrm{T} 7$ & 1 & 0,35 & - & - & 0,03 & - & - & - & - & - & - \\
\hline $\mathrm{T} 8$ & 1 & 0,35 & - & - & - & 0,03 & - & - & - & - & - \\
\hline T12 & 1 & 0,195 & 0,009 & - & - & 0,03 & - & - & - & - & - \\
\hline T18 & 1 & 0,35 & - & - & - & - & 0,03 & - & - & - & - \\
\hline P1 & 1 & 0,292 & - & - & - & - & & 0,033 & - & - & - \\
\hline P3 & 1 & 0,331 & - & - & - & - & - & - & 0,057 & - & - \\
\hline P6 & 1 & 0,306 & - & - & - & - & - & - & - & 0,129 & - \\
\hline P7 & 1 & 0,317 & - & - & - & - & - & - & - & - & 0,049 \\
\hline
\end{tabular}

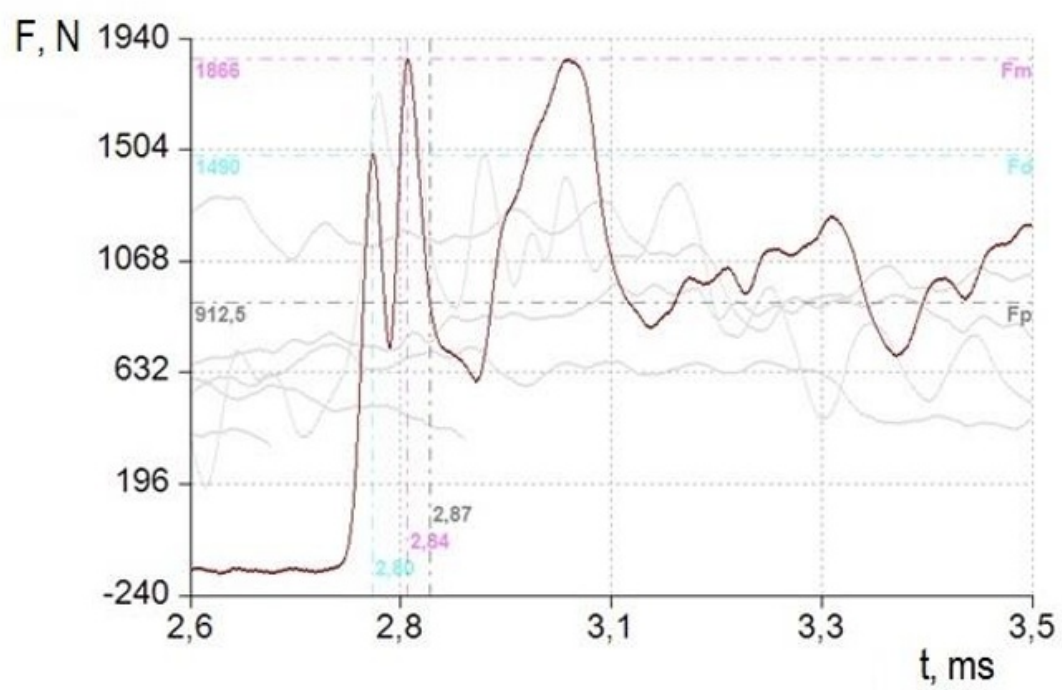

Fig. 1. The curve of the dependence of the force of the impactor on time 
According to the graphs obtained, the parameters of maximum contact force $\left(F_{\max }\right)$ and duration of contact $(t)$ were determined for the composites in question. The magnitude of the force pulse was determined from the formula

$$
J=\int_{0}^{t} f(t) \partial t
$$

where $f$ - contact force recorded during the destruction of the sample, $t$ - duration of contact between the impactor and the sample.

The calculated value of the force pulse correlates with the index of impact strength, which is estimated by the impact energy [17].

\section{Results and discussion}

For composition 1, the maximum contact force is $1866 \mathrm{~N}$, the duration of the contact is 0.13 msec, and the magnitude of the force pulse is $81.1 \mathrm{~N} \cdot \mathrm{ms}$. Further analysis of the results of studies of samples of different groups is given. Figures 2-4 show the test results. The parameters of the maximum contact force, duration of contact and impulse value for testing different compositions of cement composites are shown in graphs in relative values with respect to composition 1 .

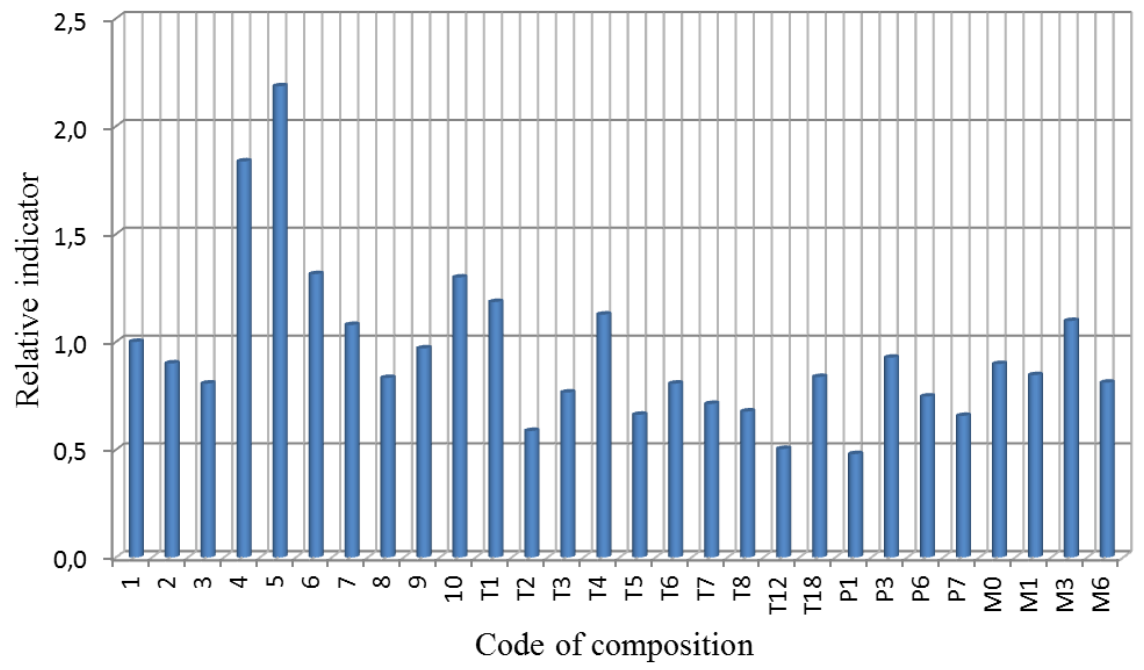

Fig. 2. Dependence of the relative change in the maximum contact force of cement composites on their composition.

Comparison of the test results shows the following. The samples on the basis of the normal density test showed that the value of the maximum contact force is higher than in the samples with an elevated water-cement ratio (composition 2) and is significantly higher than in the composition with the plasticizer (composition 3). Obviously, in this case, porosity affects. More dense materials showed a greater contact force of destruction. In this case, the duration of contact and the value of the impact of the impactor on the samples of the normal density test are also higher than those with increased water content and with the addition of a plasticizer. 
The high-filled composition including the plasticizer «Melflux 1641F» showed better results than the composite plasticized with the additive «Khidetal $9 \gamma$ » (compositions 4 and 8). At the same time, when testing the cement stone, better results were obtained for composites with a softener «Fortress-Strong».

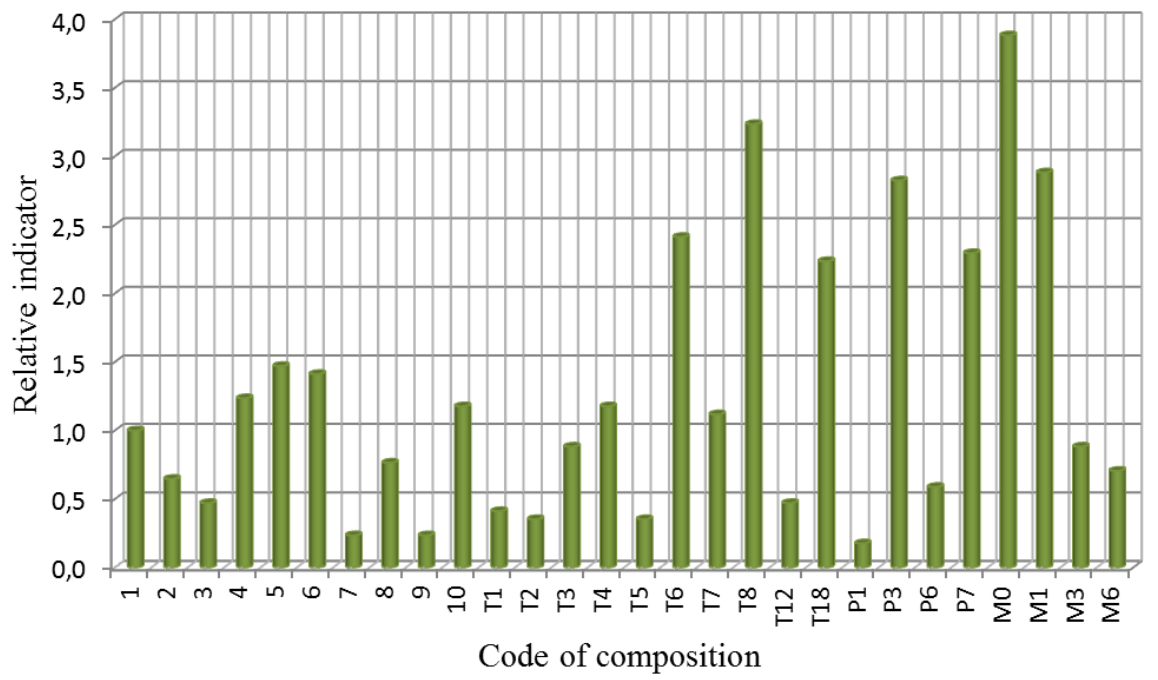

Fig. 3. Dependence of the relative change in the duration of contact between cement composites and their composition.

The introduction of sand aggregates into the compositions of cement composites led to an increase in the maximum contact force by $33.8 \%$ compared to the control composition. From this point of view, the compositions containing finely dispersed fillers, which were used as a microcrew and microsilica, are of considerable interest.

Plasticized high-filled compositions of the new generation 4 and 5 showed high values of maximum contact force, duration of contact and pulse size. The composite which includes a micro-rock quartz, quartz filler and fine aggregate, as well as a hyperplasticizer «Melflux 1641F», had a higher indicator of maximum contact force and contact time compared to compositions 6 and 7, which is characterized by higher rigidity and strength of the material. On the contrary, old-aged concretes (compositions 6 and 7) have a shorter contact time and a deformation and destruction force impulse in comparison with new and transitional generations (compositions 5 and 4, respectively).

This is due to the fact that the change in the structure of the matrix of new-generation concretes due to filling with rheologically-active dispersed powders and the binding of portlandite with microsilica provides energy dissipation in the bulk of the material due to the much higher energy of deformation and fracture compared to conventional concrete. For comparison: the maximum contact force in the 4 composition is $3808 \mathrm{~N}$, which is $84 \%$ higher than for the cement stone from the normal density test $(2073 \mathrm{~N})$. At the same time, the parameters of the duration of the contact and the value of the impulse are approximately twice as high. An even higher result was in case of introduction of microsilica into the highly-filled formulations (composition 5). The maximum contact force at which the samples were destroyed was $4530 \mathrm{~N}$, which is more than twice higher than for a cement stone based on a normal density test. 


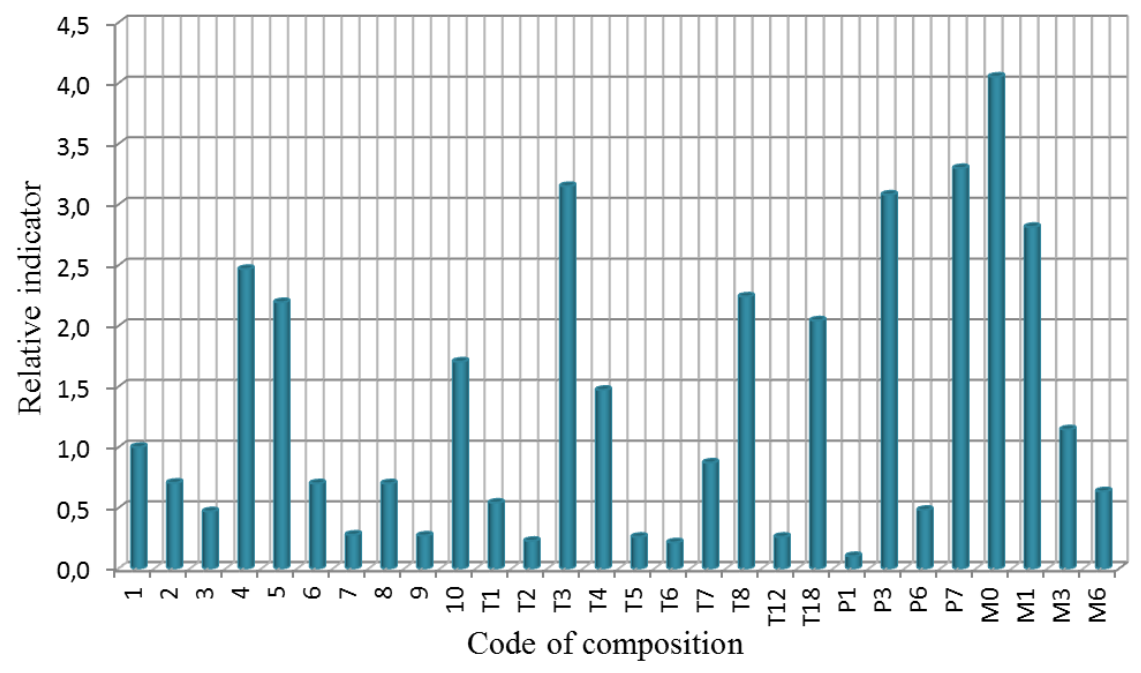

Fig. 4. Dependence of the relative change in momentum of the impactor of cement composites on their composition.

When comparing the impact strength of decorative cement composites (P1, P2, P3, P4) pigmented with iron-oxide red, iron-yellow, green glauconite, and iron oxide adopted in various quantitative contents, taking into account the hiding power, it was found that better results have compositions colored with iron-yellow and green glauconite pigments. The same compositions are characterized by a higher content of powdered pigment.

Sequential activation of the mixing water in the chambers of electrochemical activation with a current density $j_{\max }=22.58 \mathrm{~A} / \mathrm{m}^{2}$ and electromagnetic activation with an electromagnetic field strength of $H_{\max }=75 \mathrm{kA} / \mathrm{m}$ [E $\left.+\mathrm{M}(3-3)\right]$ regime resulted in an increase in the maximum contact force with a decrease in the duration of contact and the magnitude of the pulse.

In insignificant limits - an average of $10-15 \%$ - there was a decrease in the impact strength of cement stone with the addition of various bactericidal preparations: «Teflex Desinficant», «Teflex Universal», «Ultrades-Bio», «Teflex anti-mold». At the same time, the introduction of the last biocide resulted in a lesser decrease in the maximum contact force during failure. On the example of two compounds, one of which simultaneously contains the biocide «Ultrades-Bio» and hyperplasticizer «Melflux 5581», and in the other the plasticizer is absent, it is clear that the same indicators are obtained.

\section{Conclusions}

As a result of the research, high impact strength of powder-activated concrete was obtained, which can be recommended for use in structures of buildings and constructions, including underground structures of the subway exposed to shock loads.

\section{References}

1. V.I. Kalashnikov, Conc. and reinf. conc., 1, 82 (2012)

2. V.I. Kalashnikov, Techn. of conc., 1, 22-26 (2008) 
3. V.I. Kalashnikov, V.T. Erofeev, O.V. Tarakanov, News of H. Ed. Establ. B., 30-37 (2016)

4. V.I. Kalashnikov, V.T. Erofeev, O.V. Tarakanov, V.P. Arkhipov, High-Str. Cem. Conc.: Techn., Const., Econ., 36 (2016)

5. V.I. Kalashnikov, V.M. Volodin, M.N. Frost, etc., The Young Sc., 207-210 (2014)

6. V. Erofeev, V. Kalashnikov, D. Emelyanov, E. Balathanova, I. Erofeeva, V. Smirnov, I. Tretiakov, A. Matvievskiy, Solid State Phenomena, 871, 33-39 (2016)

7. V. Erofeev, V. Kalashnikov, D. Emelyanov, E. Balathanova, I. Erofeeva, O. Smirnova, I. Tretiakov, A. Matvievskiy, Solid State Phenomena, 871, 22-27 (2016)

8. E.V. Gulyaeva, I.V. Erofeeva, V.I. Kalashnikov, A.V. Petukhov, The Young Sc., 191194 (2014)

9. V.I. Kalashnikov, I.V. Erofeeva, V.M. Volodin, D.A. Abramov, Modern probl. of sc. and educ., 1-2 (2015)

10. E.V. Gulyaeva, I.V. Erofeeva, V.I. Kalashnikov, A.V. Petukhov, The Young Sc., 194196 (2014)

11. M.N. Moroz, V.I. Kalashnikov, I.V. Erofeeva, The Young Sc., 189-191 (2015)

12. E.M. Chernyshov, N.D. Potamoshneva, O.V. Artamonova, etc, B. materials, 32-36 (2008)

13. Yu.M. Bazhenov, E.M. Chernyshov, D.N. Short, B. materials, 6-14 (2014)

14. D.N. Korotkikh, D.N. Short, Crack resistance of modern cement concretes (State Agricultural Academy, Voronezh, 2014)

15. E.M. Chernyshov, D.N. Short, B. materials, equip. and tech. of the XXI c., 30-32 (2008)

16. E.M. Chernyshov, D.N. Korotkikh, O.V. Artamonova, Proc. of the Cent. Reg. Branch of RAASN, 102-123 (2010)

17. K. Reincke, W. Grellmann, A. Soever, L. Frormann, GAK 65, 5, 290-296 (2012) 